\title{
Effects of Two Experientially-Correct Introduction To Engineering Modules on Prospective Female Engineering Students
}

Dr. Jerry Volcy, Spelman College

Jerry Volcy is President of JVLabs, LLC, COO or SoftWear Automation and a part-time member of the faculty at Spelman College. JVLabs is an engineering consultancy specializing in the advanced development of FPGA designs, microprocessor microcode and O/S device drivers. SoftWear Automation is a DARPA funded startup chartered to automate the manufacture of sewn goods through robotic automation. Dr. Volcy is a graduate of the Georgia Institute of Technology and holds multiple patents in the fields of fiber optics and electro-optics. His research interests include the areas of medical and bio-embedded processors as well as in the sustainable deployment of technology in developing nations. 


\title{
Effects of Two Experientially-Correct Introduction To Engineering Modules on Prospective Female Engineering Students
}

\begin{abstract}
"Experientially Correct Introduction to Engineering" (ECIE) is a loosely defined term coined by the authors to refer to any introductory academic engineering exercise that deliberately aims to incorporate as many aspects of real-engineering as possible in order to create an experience that accurately reflects the practice of engineering. For incoming students, up to the point when they arrive on a college campus, most introductory engineering activity is intended to entice or awaken the student to the possibilities of engineering. ECIE exercises, on the other hand, aim for accuracy of practice as a counter measure to downstream attrition resulting from shifting perceptions of engineering as students progress through the curriculum. In this paper, we discuss the challenges and the effects of designing two six-week summer bridge ECIE modules for prospective female engineering students who have no prior technical training and no prior skill in the supporting trades. Details of module development philosophy including choice of activity, technical difficulty, relevance, disciplinary diversity are discussed. Effects of the modules are assessed by student survey. It is found that what students reported to be their greatest surprise about engineering based on their ECIE module experience can be generally grouped into 4 recurring themes: 1 ) Engineering can be largely trial-and-error and not necessarily math-intensive. 2) Engineering is highly demanding in terms of attention to detail. 3) Engineering can have a very large impact on society 4) Engineering can be an isolating discipline for the practitioner. Suggestions on how this insight might be used to attract and retain more female students to engineering are provided.
\end{abstract}




\section{Introduction}

At Spelman College, incoming students have the option of majoring in several STEM disciplines, including a dual-degree engineering program (DDEP) administered through a cooperative agreement with partnering universities. Consistent with the national trends, the college suffers some degree of attrition of students who start out as engineering majors but fail to persist to completion. There is also a missed opportunity of students who do not consider engineering in large part because they do not really understand what it is. Indeed, there is an abundance of evidence in the literature suggesting that incoming college students ${ }^{2,3}$, as with the broader population ${ }^{1}$, are generally ill-informed about what engineering is and what engineers do beyond a very cursory understanding.

Up to the point when they arrive on the college campus, many of the students have had limited opportunities to engage in engineering activity. Such engagements, when they are available, are typically offered extra-curricular or through outreach programs that aim to increase awareness and entice students into considering engineering as a future career option. To fulfill this objective, these programs tend to highlight the rewarding and exciting aspects of engineering and are of incalculable importance in boosting confidence, piquing student interest and sustaining an improved college bound engineering pipeline. These programs, however, do not necessarily equip the students with the complete picture of engineering needed to make a more informed decision about pursuing an engineering degree. It is the authors' thesis that this partiallyinformed condition contributes to downstream attrition as students eventually form a more complete picture of what engineering is and what success in its study and practice entails.

To address this, rising STEM freshmen admitted to the college, both engineering and non-engineering, are invited to participate in a credit-optional introduction to engineering course administered as a summer-bridge program. The central component of the program is a six-week, hands-on engineering module where students are challenged to complete an engineering project. For the past two summers, the authors (who are also the designers of the engineering modules), have adopted the objective of creating an "Experientially Correct Introduction to Engineering" (ECIE) module. ECIE is a loosely defined designation coined by the authors to refer to any introductory academic engineering exercise that deliberately aims to incorporate as many aspects of real-engineering as possible in order to create an experience that accurately reflects the study and/or practice of engineering. ECIE has the objective of accurate representation-it neither aims to entice nor dissuade, encourage nor turn students away from engineering. Rather, it aims to un-biasedly lay bare the realities of engineering study and practice in order to empower students to self-select in and out of engineer early in their college careers. This means that, for an ECIE module, not only should the fun and gratifying aspects of engineering be born out, but also the level and nature of the effort required for success must be appropriately represented (later on, we will show how, left without this guidance, many students under- and over-estimate or otherwise misjudge the type of effort required in the study and practice of engineering). 
In order to accomplish this, the module designers have identified and outlined a number of criteria-some evidence-based and some exploratory-intended to be a framework that insures that the modules deliver results that are inline with the overall ECIE objective. Defined more concisely, an ECIE module is a technically non-trivial introductory engineering problem whose solution is partially hands-on, collaborativelyobtained, multi-disciplinary and may involve other constraints typically encountered in the practice of engineering including budgetary limits, delivery timelines, process scheduling, design challenges, etc. We elaborate on this definition in the next section.

\section{ECIE Framework}

In developing the modules for the summer bridge program, the authors attempted to incorporate into the module pedagogy as many of the challenges commonly encountered during the practice of engineering as possible. While this, by definition, occurs naturally in practice, our challenge is to introduce them at a level that is consumable by a student body with no formal technical training for the purpose of empowering informed career decision-making. We capture the guidelines for ECIE as required and optional module criteria below.

\section{ECIE Essentials}

- Hands-On

The module must be hands-on, requiring students to participate at a level beyond books and class time. This requirement is in-line with studies that show that such approaches improve content retention and student attentiveness ${ }^{1,6}$.

- Relevance

The module must be relatable to the students' everyday experiences. It must address an issue or solve a problem that a student can easily extrapolate to her everyday needs or experiences (e.g., building a solar-powered car vs. building a machine that calculates Fibonacci Numbers).

- Non-Trivial

The module must present the student with a non-trivial problem. That is, the technical challenge must be such that the problem cannot be solved by intuition and trial-and-error alone (e.g., a self-steering car vs. a mousetrap car). Rather, these projects demand grasping and applying engineering concepts (e.g.: pulsewidth modulation, micro-controller programming, etc.) beyond what is garnered through life experiences.

- Cross-Disciplinary

Engineering is a broad field comprised of many sub-disciplines (chemical, electrical, mechanical, civil etc). Additionally, solving engineering problems often requires inclusion of other specialties (computer science, mathematics, physics, 
optics etc...). While no single module can capture aspects of all these inter-related disciplines, ECIE is deliberate in its attempt to include as many of these as possible.

- Vertical Diversity

Engineering is also practiced at many levels, from the technician to the researcher. The module aims to engage student at multiple levels, including the technician level, the design level and the research level.

- Collaborative Learning

As is true in practice, most large engineering efforts are a collaborative effort of peers. In the same way, ECIE calls for students to work in groups. This not only reflects true engineering, but it is also a proven pedagogically effective instrument ${ }^{4,5}$. Further, the technical language required to address the problems posed by the technically non-trivial nature of the project drives collaboration in a manner that exceeds what is required on a non-ECIE project.

- Zero Pre-requisites

Despite the "non-trivial" requirement, the module must operate within the capacity of newly graduated high school student with no prior technical training. Use of standard engineering tools (oscilloscopes, MathCAD, spectrometers, CAD systems, spectrum analyzers, etc.,) must be limited to what can be learned and effectively used within the module's six-week duration.

\section{ECIE Optional Attributes}

Beyond the requirements outlined above, engineering projects are frequently characterized by several other requirements and constraints, including budgetary constraints, delivery timelines, functional specifications, empirical analysis, prototype and model development, reliability testing and many others. While it is not possible to capture all of these (indeed, only the largest engineering projects entail them all), inclusion of some of these principles either in discussion or as part of the module is desired.

\section{Two ECIE Module Case Studies}

In 2012, ten students enrolled in the summer bridge program and were tasked to build a servoed laser system that traced patterns on a wall. When told that this guided laser technology is, in principle, similar to the technology used in Lasik surgeries, student interest, as judged by classroom engagement and questions, increased noticeably. This 
observation from past experiences drives the ECIE "relevance criteria" and is consistent with other findings that show that, compared to male students, for female students, solving a technical challenge as a means to a social end has significantly more appeal than solving a similar challenge without an immediate beneficial application. Figure 1 below shows some details of the module ${ }^{6}$. Not shown in the figure is the microcontroller and support electronics to drive the servo motors and the laser diode. This circuitry was built partially on a breadboard and partially by soldering based on a provided schematic diagram. Students had to acquire the skills to read the schematic and the skills to properly assemble the circuit. This was then followed by a greater technical challenge of learning how to program the micro-controller to properly control the laser. By ECIE prescription, this module could not be solved by trial-and-error or intuition alone. It required collaborative effort that spanned several disciplines and satisfied most of the conditions the authors prescribed to be deemed an ECIE activity. Details of the project can be found in reference 6. A few long-exposure images of some of the patterns the students successfully traced with this system is shown in Figure 1.
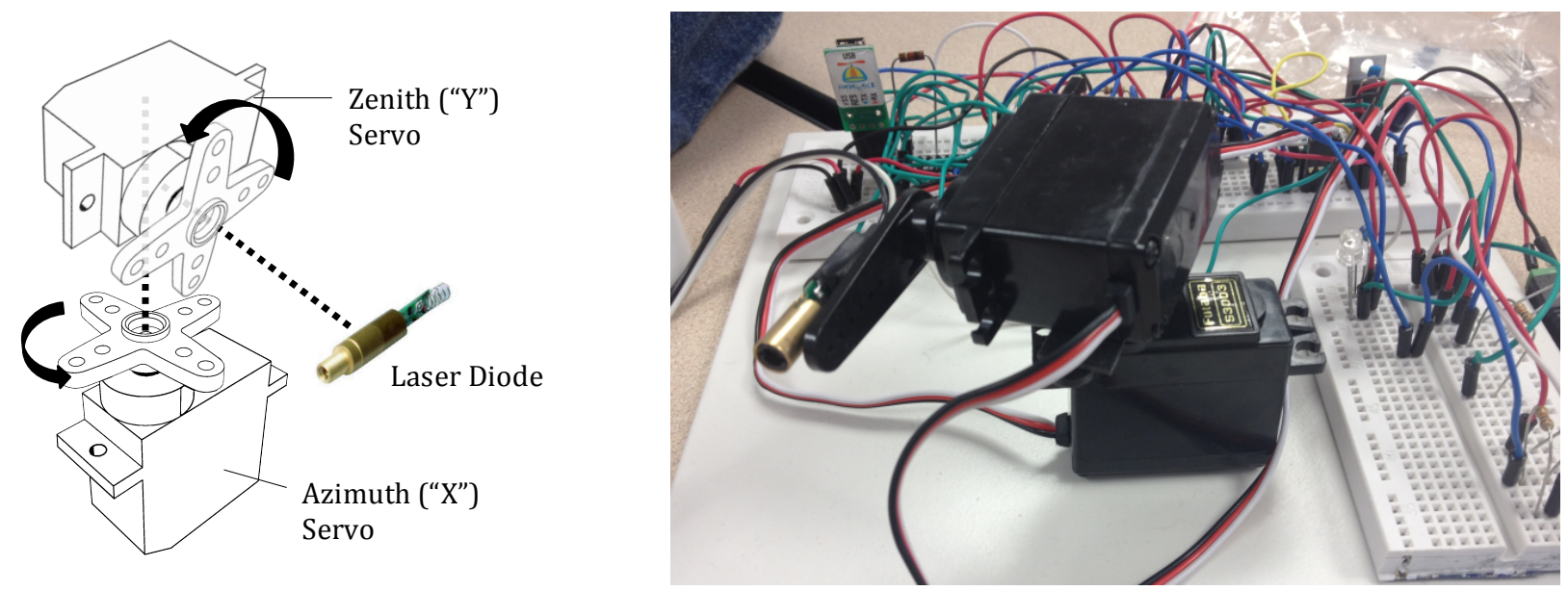

Figure 1: The figure above is an assembly drawing of the servoed laser mechanics showing a laser diode carried by a servo "zenith" motor mounted on the horn of a second "azimuth" motor. The figure in the upper right is a photograph of the apparatus showing typical circuitry for the MCU required to support the operation of the laser system. The pictures to the right are long-exposure photographs of example patterns traced out by the servoed lasers. In each case, exposure times are on the order of $0.5-3$ seconds.

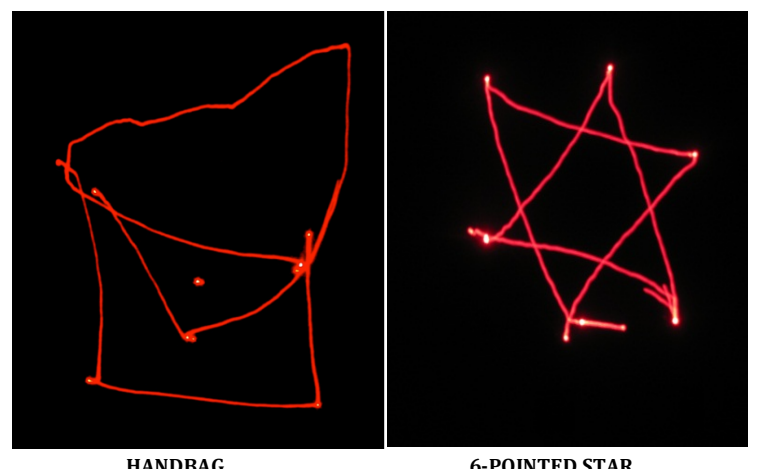

HANDBAG

6-POINTED STAR 
In 2013, ten students enrolled in the summer bridge program and were tasked to build a "SmartCar". When told they would build a self-steering, self-parking car that could one day enable the young and elderly to become independent (youngsters could take themselves to the ER, traffic jams could be greatly reduced, the elderly could take themselves to the pharmacy, etc), student interest as judged by classroom engagement, seemed to increase sharply. As before, this was an expected reaction. Refer to Figure 2 below for a few details on the module The chassis of the SmartCar is a standard 1:16 scale model radio control $(\mathrm{R} / \mathrm{C})$ car purchased from a local toy store. The $\mathrm{R} / \mathrm{C}$ vehicle is extensively modified by the students. The radio circuitry is removed and the vehicle is retrofitted with an onboard micro-controller and peripheral sensors that enable it to operate autonomously. As with the 2012 project, the students work collaboratively to acquire the technical skills needed to read the provided schematics, solder and breadboard the needed components and program the micro to carry out a specified set of tasks. Because of the limited 6week duration of the module, only two tasks were required: autonomous parallel parking and autonomous steering. These are illustrated in the figure. As with the 2012 servoed laser project, the SmartCar project sufficiently satisfied the conditions the authors deemed necessary for an ECIE

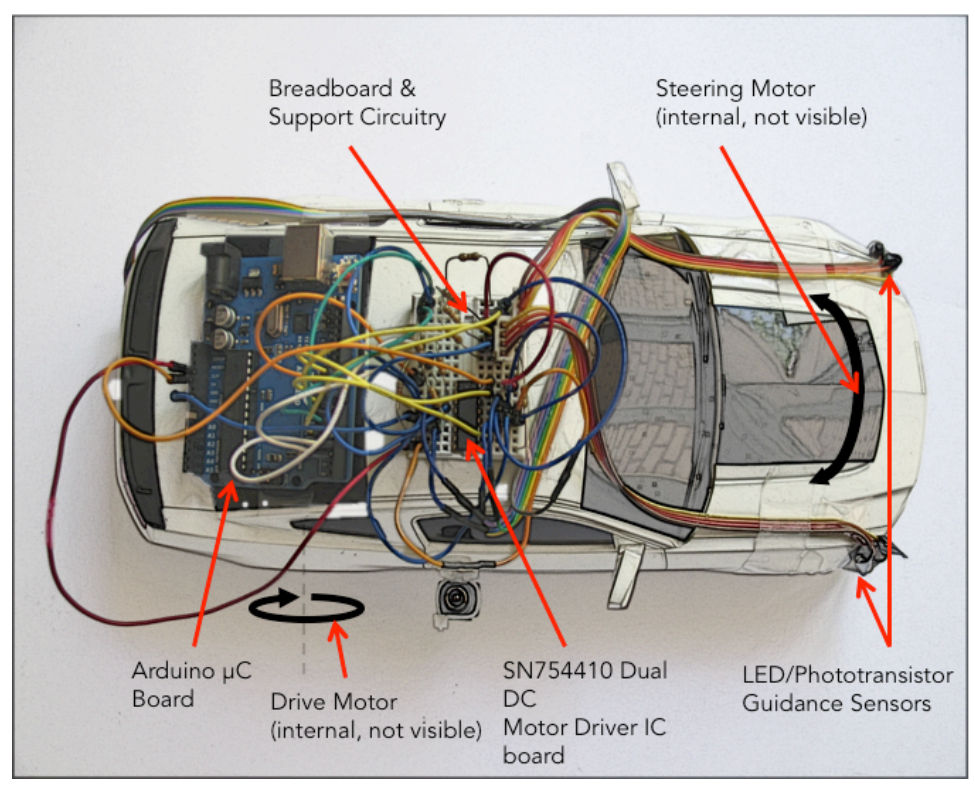
module.

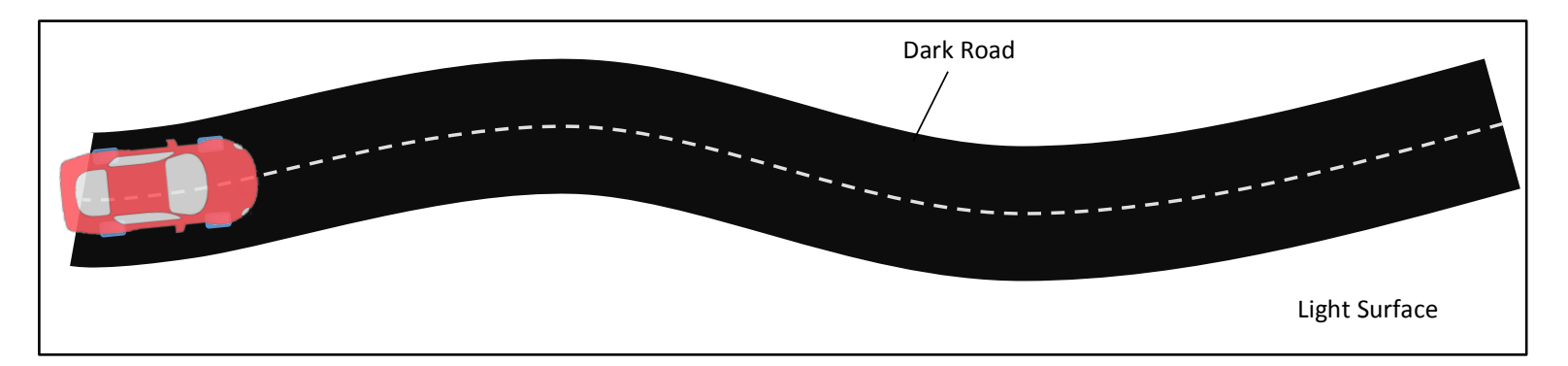

Figure 2: The photograph in the upper right shows a completed SmartCar, which is a student-modified R/C vehicle. Major components including the micro controller board programmed to control the vehicle are shown. The figure in the middle is a pictorial depiction of one autonomous task programmed by the students: in this case, self-parallel parking. The bottom image shows the final task of auto-navigation based on optical sensory feedback. 


\section{Effects of the ECIE Modules On Student Perception}

Upon completion of the modules, student migration patterns in and out of engineering was assessed. While the majority of the students who were initially either considering or not considering majoring in engineering did not change their minds, 3 of the 20 covered by this study did. Two who were initially intent on majoring in engineering decided not to pursue an engineering major while one who was initially not intent on majoring in engineering switched to engineering. This was not an entirely unexpected outcome as students gained greater understanding of what possibilities engineering provides and greater understanding of the level and nature of the effort required to succeed in the discipline.

In an attempt to evaluate the efficacy of the ECIE approach, the 2012 students were asked the following exit survey questions:

\footnotetext{
"What did you find most interesting about your intro-to-engineering experience?" and

"Was the engineering module what you expected? Explain."
}

Five of the ten students responded to at least one of these questions with a statement in the form

"I was surprised that...".

Based on this, the authors modified the 2013 exit survey to instead ask the single explicit question of

"What did you find most surprising about engineering?

The combined answers to these and other survey questions reveal four recurring thematic impressions that were insightful to the authors/course designers. For the purpose of summarization, the authors have paraphrased them and somewhat subjectively assessed them as "positive" or "negative" impressions of engineering based on the greater context of daily contact with the students. This categorization does not affect the validity of the finding. Each is described below:

\section{1) Engineering can be largely trial-and-error and not necessarily math-intensive.}

The most frequently stated ECIE feedback received from the survey was a relief expressed by many students that building a complex engineering system to solve a real problem did not entail solving complex mathematical equations. The authors judge this 5-times repeated feedback as a positive experience. This statement comes across as a clarification to a misconception about the practice of engineering and suggests a possible cause why many students do not even consider a career in engineering. Indeed, based on this finding, one student initially not an engineering major switched to engineering. Almost paradoxically, the modules are designed to be un-solvable by intuition and trial-and-error alone. Still, as is true in practice, a significant component of engineering (in product development, for example) is based on intuition and trial-and- 
error coupled in appropriate measure with scientific and mathematical principles. One possible conclusion from this observation is that the students misjudged the balance of these two. Another possible conclusion is that the students simply misunderstand that while the study of engineering does involve a good bit of mathematics in order to properly grasp the fundamental scientific principles used by engineers, the intensity and rigor of this mathematical training usually does not carry into practice. The majority of practicing engineers do not engage in solving complex mathematical problems on a daily basis as some students appear to believe. Such a misconception, if it exists widely, could certainly dissuade entry into the discipline, suggesting that an effort to better represent the norm might be an aid to recruitment.

\section{2) Engineering is highly demanding in terms of attention to detail.}

The authors judge this 4-times repeated comment as a slightly negative to neutral observation born from frustration as the students worked to complete their modules. When asked to elaborate, the comment seems to reflect the frustration of getting every sub-system to work just right in order for the whole to operate properly. Exact comments include "It all seems precarious" and "I don't see how you can make anything reliable like this" and "it's just like math when you get the whole problem wrong for a little mistake" and "It's too tedious".

Following the 2012 ECIE module, one student originally intent on majoring in engineering changed her mind and provided the explanation that engineering is "too tedious" as her reason for doing so. The authors believe that this repeated observation by the students is a fair assessment of engineering and highlights the ECIE objective of accurately presenting, not just the level, but the nature of the effort required for success in engineering. While students are often pre-conditioned to the idea that "engineering is hard", lacking any qualifier, this notion is not useful in assessing one's penchant for the discipline. The statement sheds no light on the nature of the required effort. It is a central part of the ECIE principle that a student who comes to realize the nature of engineering early on is more desirable than one who comes to the same conclusion after two years of training.

\section{3) Engineering can have a very large impact on society}

A very positive comment, particularly from the 2013 SmartCar module participants, is a newly gained appreciation for engineering's potential to dramatically improve the standards of living. This appreciation was expressed even by students who chose not to pursue an engineering degree. Somewhat serendipitously, throughout the SmartCar module, there was a fair amount of press about self-driving vehicles, that made for lively class discussions about how such vehicles could impact society. One such discussion focused on how a self-driving vehicle could allow a single parent to commute to work while simultaneously sending her child to school. Another touched on the promise of coupling the technology with GPS to eliminate the need for traffic lights, stop signs, painted roadways, highway signs and a myriad of other expenses that could be used to, say, improve schools and lower taxes. These spontaneous discussions of hypothetical 
cascading benefits of a SmartCar resonated extremely well with the students and provided a sense of how important the work they were doing-and engineering in general-could potentially be.

The authors found it surprising that a plurality of students identified engineering's potentially large societal impact a their "biggest surprise" since most of the students who claim so provided reasonably good answers to another survey question asking them to define engineering. This suggests that, as is the case in many situations, while in principle, these students understand what engineering is, until they reflect (through engagement or explicit direction) on the discipline, the full realization of the definition of engineering and its full implications may not be realized. This would further suggest that at the pre-college level where career options are evaluated, more attention be paid to exactly how personal (cell phones, PDA, Internet service, etc.) and far-reaching engineering activity actually is.

\section{4) Engineering can be an isolating discipline for the practitioner.}

At the start of the course, (particularly in the dis-assembly phase of the projects), many of the students enthusiastically took photographs of what they were working on and shared them through social media with family and friends. This was notably an important activity for one first-generation college attendees enrolled in 2012. By the end of the first week however, the material becomes sufficiently abstract that such communications to the lay public becomes difficult. By the end of the second week, the concepts the students are working with and the vocabulary required to do so, begins to isolate them from colloquial conversation and it is no longer possible to convey the more interesting details about their activity without extensive explanations. This recurring observation arose not from the question of "biggest surprise" as did the others, but from a separate survey question asking the students to state their least favorite part of their summer experience. Nonetheless, its 3-time recurrence warrants its inclusion as a recurring student experience. The authors judged this experience as slightly negative for the students.

Perhaps just as importantly as what the students reported to be their biggest surprise, is what they did not report. Despite investing long (and often frustrating) hours to complete a challenging module, none of the students reported to be surprised by the difficulty of the material. This perhaps reflects an expectation that for some students exceeds the reality of the difficulty of the discipline. If so, this too could potentially keep otherwise capable candidates away from considering engineering. 


\section{Conclusion}

ECIE modules aim to offer students the opportunity to experience engineering in a way that empowers them to make informed decisions about whether or not to pursue an engineering degree. In this paper, we show that the an accurate exposure to engineering can serve to eliminate misconceptions and provide clarity about engineering that is important in the process of selecting and persisting in the major. Further, recurring themes from students who complete the ECIE modules offer some insights that may be useful in refining tactics for recruitment of additional female students into the discipline.

\section{References}

[1] Committee on Public Understanding of Engineering Messages, Changing the Conversation: Messages For Improving Public Understanding of Engineering, The National Academies Press, Washington, D.C. , 2008.

[2] Cunningham, C., and Knight, M., "Draw an Engineer Test: Development of a Tool to Investigate Students' Ideas about Engineers and Engineering". Proceedings of the 2004 American Society for Engineering Education Annual Conference and Exposition. Salt Lake City, Utah, June 20-23. ASEE, Washington, D.C., 2004.

[3] Cunningham, C., C. Lachapelle, and A. Lindgren-Streicher., "Assessing Elementary School Students Conceptions of Engineering and Technology". Proceedings of the 2005 American Society for Engineering Education Annual Conference and Exposi tion. Portland, Ore., June 12-15. ASEE, Washington, D.C., 2005.

[4] Peercy, P., Cramer, S., "Redefining Quality in Engineering Education Through Hybrid Instruction", Journal of Engineering Education, October 2011, Vol. 100, No. 4, pp. 625-629.

[5] Stump, G., Hilpert, J., Husman, J., Chung, W., Kim, W., “Collaborative Learning in Engineering Students: Gender and Achievement", Journal of Engineering Education, July 2011, Vol. 100, No. 3, pp. 475-497.

[6] Volcy, J., Sidbury, C. K. "Developing a Summer Bridge Course for Improving Retention in Engineering". Proceedings of the ASEE 120th Annual Conference, June 23-26, 2013, Atlanta, GA. 